\title{
CORRECTIONS
}

\section{ESTIMATION OF THE CORRELOGRAM FOR A STATIONARY GAUSSIAN PROCESS BY RANDOM CLIPPING}

\author{
By MINORU TANAKA
}

Vol. 9, No. 3, pp. 385-400 (1986).

The author discovered an error in Theorem on page 388 after the article had gone to press: The second term $\mu^{2}(2 t+1)^{2}\left[\left(\sigma^{2}+1\right)^{2}-t^{2}\right]$ of the numerator of $I_{1}\left(\mu, \sigma^{2} ; x\right)$ on page 388 should be $\mu^{2}(2 t+1)^{2}\left(\sigma^{2}+1-t\right)$. This is because an error occurs in the formulation (A.6) on page 396: The denominator $\left(\sigma^{2}+1+x\right) \sqrt{\left(\sigma^{2}+1\right)^{2}-x^{2}}$ of the second integral of the middle part of (A.9) should be $\left(\sigma^{2}+1+x\right)^{2} \sqrt{\left(\sigma^{2}+1\right)^{2}-x^{2}}$. The correct expression necessitates the following changes to the numerical computations of Tables $1-5$ in Section 3 :

Page 389

Table 1: The tenth row of the fourth column $\left(\operatorname{Var}\left[\gamma_{1}^{(1)}\left(\mu^{*}, \sigma^{* 2}\right)\right]\right)$ should be $0.1000(0.0221)$. The vectors $\left(\mu^{*}, \sigma^{* 2}\right)$ at $\left|\rho_{1}\right|=0.6,0.7,0.8$ and 0.9 should be $(0.2,0),(0.1,0),(0,0.05)$ or $(0.2,0)$ and $(0.3,0)$.

\section{Page 390}

Table 2: The upper part of the fifth column up to $h=4$ should be 0.0462 (0.0099), $0.0439(0.0092), 0.0422(0.0085)$ and $0.0449(0.0088)$. The sixth column should be $(0.3,0.02),(0.3,0.02),(0.3,0.02),(0.3,0.03),(0.5,0.02),(0.8,0.03),(0.6$, $0.02),(0.4,0),(0.4,0),(0.4,0),(0.7,0.02),(0.6,0.01),(0.7,0.02)$ and $(0.7,0.02)$.

Page 391

Table 3: The upper part of the fifth column up to $h=6$ should be 0.0018 (0.0003), $0.0906(0.0218), 0.0116(0.0024), 0.1032(0.0247), 0.0276(0.0059)$ and 0.1110 (0.0263). The vectors $\left(\mu^{*}, \sigma^{* 2}\right)$ at $h=5,6,25$ should be $(0.4,0)$ and at $h=20$ should be $(0.5,0)$.

Page 392

Table 4: The vectors $\left(\mu^{*}, \sigma^{* 2}\right)$ at $h=1,3$ and 4,6 should be $(0,0.01)$ or $(0.1)$ and $(0.2,0)$.

Page 393

Table 5: The upper part of the fifth column up to $h=6$ should be 0.0480 
(0.0100), $0.0572(0.0118), 0.0687(0.0141), 0.0810(0.0165), 0.0933(0.0190)$ and 0.1043 $(0.0213)$. The vectors $\left(\mu^{*}, \sigma^{* 2}\right)$ up to $h=9$ should be $(0.3,0.04),(0.3,0.04),(0.3$, $0.04),(0.3,0.03),(0.3,0.03),(0.4,0.03),(0.5,0.03),(0.6,0.04)$ and $(0.7,0.04)$. 
